\title{
BACK TO BASICS
}

By prioritizing research on potential bioweapon agents, the $\mathrm{NIH}$ is diverting vital resources away

from basic research on microorganisms that have an ongoing impact on public health.

Placing the United States (US) in a pecking order of nations that successfully foster an environment allowing the advancement and application of biology is not difficult. They are rightfully ranked at the top of such a list. The US government organization that has overseen this global pre-eminence in biological research is the National Institutes of Health (NIH), and their investment in basic research on microbial biology has made possible extraordinary advances in all areas of science and public health. Their far-sighted approach to support basic research has paid for itself many times over, to the extent that a whole industry is built on and exploits the discoveries made from NIH-funded basic research into microorganisms. However, a decision taken in 20012002 to prioritize research with potential bioweapon significance threatens to undermine the impact of NIHsupported microbiological research. Indeed, recent funding patterns relating to microbial physiology, genetics and pathogenesis suggest that the commitment of $\mathrm{NIH}$ to basic research is being compromised and, if allowed to continue, will undermine US public health and national interests and could have long-lasting repercussions for the future of microbiological research.

Following the anthrax attacks in 2001, there has been a huge influx of funding, institutions and investigators into research that focuses on a limited number of microorganisms that have the potential to be used as bioweapons. The species prioritized by the NIH's National Institute for Allergy and Infectious Diseases (NIAID) - those microorganisms that cause anthrax, brucellosis, glanders, melioidosis, plague and tularaemia - are responsible for, on average, zero deaths per year in the US. But the number of grants awarded by the NIAID between 2001 and 2004 that reference these agents has increased 15-fold when compared with the previous 4-year period. In isolation, this input of resources could be perceived as a welcome development in response to the realities of the dangerous times we live in. However, it seems that there is a price to pay for focusing on a handful of organisms with bioterror threat potential. Over the same period, the number of grants awarded to study non-biodefence-related pathogens has decreased by $27 \%$, despite the fact that these agents are responsible for thousands of cases of illness and deaths each year, while grants for studying model bacteria such as Escherichia coli have fallen by a massive $41 \%$. These compelling statistics, derived from the NIH's own data, form the basis of an unprecedented protest that is being taken by over 750 leading microbiologists in the US. In an open letter to Elias Zerhouni, Director of the NIH, these researchers point out that the agency's emphasis on biodefence has diverted crucial resources away from basic research, at a time when there is exceptional potential for breakthroughs that could have significant public health benefits - advances that would also have a major impact on our understanding of bioweapon agents and the development of effective therapies to control them ${ }^{1}$.

The message of this protest action is clear: to successfully develop vaccines and treatments against microorganisms that usually have little public health significance, are poorly characterized and are experimentally less tractable, it is vital that the proper level of resources is made available to study the biology of model microorganisms. Advances in our understanding of these organisms can often be immediately applied to the prevention and control of infectious diseases with important public health ramifications and ultimately to those few bacteria and viruses that could be used in bioterrorism. Failure to address the issue of research funds being diverted to projects of high biodefence importance but with little public health relevance represents, in the words of the letter's signatories, "a misdirection of NIH priorities". The price of failure to take corrective action is worrying, and places the NIH and US at risk of missing research opportunities that could have a considerable positive impact on public health and economic development.

1. Altman, S. et al. An open letter to Elias Zerhouni. Science $\mathbf{3 0 7}$, 1409-1410 (2005) 